\title{
Inactivation of the sfgtr4 Gene of Shigella flexneri Induces Biofilm Formation and Affects Bacterial Pathogenicity
}

\author{
Abdelmoughit Kaoukab-Raji ${ }^{1}$, Latéfa Biskri ${ }^{1,2,3,+}$ - and Abdelmounaaïm Allaoui ${ }^{1,2,4, *, \ddagger}$ \\ 1 Laboratoire de Bactériologie Moléculaire, Faculté de Médecine, Université Libre de Bruxelles, \\ Route de Lennik, 808, 1070 Brussels, Belgium; abdelmoughit.kaoukab@gmail.com (A.K.-R.); \\ latefa.biskri@um6p.ma (L.B.) \\ 2 Laboratoire de Microbiologie Moléculaire, CIPEM (Centre de Coalition, Innovation, et de prévention des \\ Epidémies au Maroc), Mohammed VI Polytechnic University (UM6P), Lot 660, Hay Moulay Rachid, \\ 41130 Benguérir, Morocco \\ 3 Medical Application Interface Center (CIAM), Mohammed VI Polytechnic University (UM6P), \\ 43150 Benguérir, Morocco \\ 4 Microbiome and African Genome Center (AGC), Agrobiosciences department Mohammed VI Polytechnic \\ University (UM6P), Lot 660, Hay Moulay Rachid, 41130 Benguérir, Morocco \\ * Correspondence: abdelmounaaim.allaoui@um6p.ma; Tel.: +212-5-25072926 \\ + Current Address: Affiliations 2 and 3. \\ $\ddagger$ Current Address: Affiliations 2 and 4 .
}

Received: 7 April 2020; Accepted: 21 May 2020; Published: 4 June 2020

check for updates

\begin{abstract}
Biofilm formation is a significant cause for the environmental persistence of foodborne pathogens. This phenomenon remains misunderstood in Shigella flexneri whose pathogenicity is mainly associated with the virulence plasmid pWR100. Sequence analysis of the latter predicts a putative lipopolysaccharides (LPS) glycosyltransferase (Gtr) encoded by Sfgtr4, which is the second gene of the SfpgdA-orf186-virK-msbB2 locus. We demonstrated here that purified SfGtr4 exhibited a Gtr activity in vitro by transferring glucose to lipid A. To establish the role of SfGtr4 in virulence, we generated a Sfgtr4 mutant and assessed its phenotype in vitro. Sfgtr4 mutant significantly reduced HeLa cells invasion without impairing type III effectors secretion, increased susceptibility to lysozyme degradation, and enhanced bacterial killing by polymorphonuclear neutrophils (PMNs). SfGtr4 is related to proteins required in biofilm formation. We established conditions whereby wild-type Shigella formed biofilm and revealed that its appearance was accelerated by the Sfgtr4 mutant. Additional phenotypical analysis revealed that single SfpdgA and double SfpgdA-Sfgtr4 mutants behaved similarly to Sfgtr4 mutant. Furthermore, a molecular interaction between SfGtr4 and SfPgdA was identified. In summary, the dual contribution of SfGtr4 and SfPgdA to the pathogenicity and the regulation biofilm formation by $S$. flexneri was demonstrated here.
\end{abstract}

Keywords: Shigella pathogenicity; glycosyltransferase; biofilm formation; cell invasion; lysozyme degradation; T3 secretion; PMNs killing

\section{Introduction}

Shigella flexneri is a Gram-negative pathogenic bacterium that causes human bacillary dysentery. Its ability to enter epithelial cells is conferred by a $214-\mathrm{kb}$ pWR100 plasmid encoding a Type 3 secretion apparatus (T3SA) [1]. A designated entry region of 30-kb of this plasmid encodes the Mxi and Spa proteins known to be involved in T3SA assembly, including the: (i) translocators IpaB, IpaC, and IpaD; (ii) effectors (IcsB, IpgB1, IpgD, and IpaA); (iii) chaperones (IpgA, IpgC, IpgE, and 
Spa15); and (vi) transcription activators VirB and MxiE (reviewed in [2]). Plasmid pWR100 also contains several genes encoding proteins required for bacterial cell-to-cell spread, such as IcsA [2]. Despite the significant progress made to unravel the content of pWR100, to date, several genes remain uncharacterized. In the present work, we investigated the function of orf186, the second gene located in the SfpgdA-orf186-virK-msbB2 locus. The first three genes share 93\% DNA sequence identity with shf, capU, and virK of Escherichia coli (EAEA 042) [3].

The equivalent of SfPgdA in Gram negative bacteria includes E. coli's PgaB and Yersinia pestis' HmsF, both of which are required in biofilm development [4,5]. The lipoprotein PgaB is known to introduce a limited amount of glucosamine into the poly- $\beta-1,6-N$-acetyl-D-glucosamine (PGA) polymer and therefore facilitate its export via the porin PgaA [5]. HmsF possesses a polysaccharide deacetylase activity [4] and contains the COG1649 domain, required for biofilm development [6]. Up to date, the closest well-characterized counterpart to both SfPgdA and Shf is the IcaB protein found in Staphylococcus epidermidis, which is also known to be involved in biofilm formation [7]. IcaB is a secreted protein involved in the mechanism of intercellular adhesion but also acts as a poly-N-AcGlc deacetylase of the exopolysaccharide (EPS) [8].

It is well known that planktonic cells produce EPS in response to a variety of environmental signals such as magnesium deficit and subsequently facilitating bacterial attachment [9]. Optimal expression of the SfpgdA-orf186-virK-msbB2 locus is associated with low $\mathrm{Mg}^{2+}$-containing media and is regulated by the PhoP/PhoQ two-regulatory component system [10,11]. In a previous study, we reported that Shigella strains lacking either PhoP or SfPgdA are highly susceptible to lysozyme degradation and are less resistant to killing by polymorphonuclear neutrophils (PMNs) [12].

Sequence alignment revealed that orf186 of S. flexneri, encodes a predicted LPS glycosyltransferase 4 (Gtr4). Orf186, renamed here SfGtr4 (for S. flexneri Gtr), shares 73\% sequence identity with RfbU of E. coli [13]. RfbU is also involved in LPS biosynthesis and contains a RfaG domain associated with biofilm formation [14]. Gtrs catalyze monosaccharides transfer from an activated donor, such as a sugar-nucleotide, to an acceptor molecule [15]. Gtrs may therefore transfer UDP-, ADP-, GDP-, or CMP-sugar from activated donor molecules to specific acceptor molecules (e.g., lipid, protein, heterocyclic compound, or carbohydrate residue), reflecting a wider range of biological functions (CAZy database, http://www.cazy.org/GT1_bacteria.html).

Here, we sought to determine whether biofilm formation occurred in Shigella and to establish if Sfgtr4 is involved in such process. We found that biofilm formation was increased by the deletion of Sfgtr4 gene, and phenotypical analysis revealed that Sfgtr4 and SfpdgA are both required to fulfill Shigella infection of HeLa cells or PMNs.

\section{Material and Methods}

\subsection{Bacterial Strains and Growth Conditions}

Strains used in this study are derivative from the wild-type Shigella flexneri 5 strain M90T-Sm [16] (Table 1). Bacteria were grown in tryptic casein soy broth (TSB, Becton Dickinson, Belgium) at $37^{\circ} \mathrm{C}$. E. coli strain DH5 $\alpha \lambda$ pir was used for the propagation of plasmids carrying an oriT origin of replication (pSW23T) [17], and SM10 $\lambda$ pir was used to transfer derivatives of pSW23T to S. flexneri. E. coli Top10 (Invitrogen, Carlsbad, CA, USA) was used for recombinant proteins production. E. coli strains were grown in Luria-Bertani (LB) medium (Becton Dickinson, Belgium) supplemented with appropriate antibiotics: ampicillin, $100 \mu \mathrm{g} \mathrm{mL}{ }^{-1}$; kanamycin, $50 \mu \mathrm{g} \mathrm{mL} L^{-1}$; streptomycin, $100 \mu \mathrm{g} \mathrm{mL}^{-1}$ and chloramphenicol, $25 \mu \mathrm{g} \mathrm{mL} \mathrm{m}^{-1}$ (VWR, France). 
Table 1. Plasmids and strains used in this study (TS)

\begin{tabular}{ccc}
\hline Plasmid & Characteristics & Reference \\
\hline pKA1 & pTZ18R encoding native SfPgdA & {$[12]$} \\
pKA2 & pTZ18R encoding native SfGtr4 & TS \\
pKA85 & pTZ18R encoding native SfPgdA and SfGtr4 & TS \\
pKA4 & pSW23T-SfpgdA::aphA-3 (Suicide vector) & {$[12]$} \\
pKA8 & pSW23T- Sfgtr4::aphA-3 (Suicide vector) & TS \\
pKA121 & pSW23T- SfPgdA -Sfgtr4::aphA-3 (Suicide vector) & TS \\
pKA20 & pMALCRI expressing MBP-SfPgdA & TS \\
pKA22 & pQE60 expressing SfPgdA-His6 & TS \\
pKA23 & pQE60 expressing SfpgDA-His6 & TS \\
\hline Strain & Genotype & Reference \\
\hline M90T-Sm & Derivative of wild-type Shigella flexneri strain M90T & {$[16]$} \\
BS176 & M90T-Sm pWR100 cured strain & {$[18]$} \\
SF401 & M90T-Sm mxiD (mxiD::aphA-3) & {$[19]$} \\
SBkr1 & M90T-Sm SfpgdA (SfpgdA::aphA-3) & {$[12]$} \\
SBkr2 & M90T-Sm Sfgtr4 (Sfgtr4::aphA-3) & TS \\
SBkr3 & M90T-Sm SfpgdA Sfgtr4 (SfpgdA-Sfgtr4::aphA-3) & TS \\
PhoP & M90T-Sm phoP (phoP::aphA-3) & {$[12]$} \\
\hline
\end{tabular}

\subsection{Construction of Single sfgtr4 and Double sfpgdA-sfgtr4 Mutants}

Upstream and downstream regions of SfpgdA and Sfgtr4 genes were PCR-amplified from plasmid pWR100. Plasmids and primers used in this study are listed in Tables 1 and 2. Primers contain restriction sites used for DNA cloning. The amplified DNA fragments, containing a BamHI site at the $5^{\prime}$ end and a SalI site at the $3^{\prime}$, were subsequently cloned into the corresponding sites of the pTZ18R vector to generate pKA2 and pKA85 plasmids. pKA2 was used to generate plasmid pKA5 by inserting the 850-bp SmaI DNA fragment carrying the $a p h A-3\left(\mathrm{Km}^{\mathrm{R}}\right)$ gene [20] into the unique EcoRV site of pKA2. Plasmid pKA85 was used to generate pKA120 by replacing the internal NruI-EcoRV DNA fragment of pKA85 by SmaI digested aphA-3 gene. SfpgdA was interrupted at codon 120 while Sfgtr4 was interrupted at codon 53. Next, an inactivated copy in each construct was inserted into the suicide vector pSW23T to generate pKA8 (Sfgtr4::aphA-3) and pKA121 (SfpgdA-Sfgtr4::aphA-3). The resulting plasmids were next transferred to $S$. flexneri M90T-Sm by conjugal mating and the structures of the two Sfgtr4 and SfpgdA-Sfgtr4 mutants were further confirmed by PCR. Plasmids pKA2 (SfGtr4), pKA1 (SfPgdA) [12], and pKA85 (SfPgdA and SfGtr4) were used in the complementation experiments.

Table 2. List of primers used in this study.

\begin{tabular}{ccc}
\hline Name & Primers 5' $^{\prime} \mathbf{3}^{\prime}$ & R.S \\
\hline sfpgdA.1 & CGGGATCCTTTAAACGAAGGGGGCATTTTG * & BamHI \\
sfgtr4.1 & CGGGATCCGGAAAGTTGCGCATGGCTG & BamHI \\
sfgtr4.2 & ACGCGTCGACCCGATAAATGATAAGTTACTTAC & SalI \\
sfgtr4his-s * & GTCCATGGGATTAAACGAAGGGGGCATTTT & NcoI \\
sfgtr4his-as ** & GCTAGATCTCATCCGGTAATCTTGGCCCC & BglII \\
sfpgdAmal-s & CGGGATCCTTAAACGAAGGGGGCATT & Bam HI \\
sfpgdAmal-as & AACTGCAGTTAATCATCCGGTAATCTTGGC & PstI \\
sfpgdAhis-s & GTCCATGGGAAATATACTATTTACGGAATCAT & NcoI \\
sfpgdAhis-as & GCTAGATCTCTTGTGCTTCGCTAATGTGAG & BglII \\
\hline
\end{tabular}

R.S: Engineered restriction sites are underlined. ${ }^{*} \mathrm{~s}$ : sense and ${ }^{* *}$ as: antisense.

\subsection{Proteins Secretion and HeLa Cells Invasion}

Crude extracts and concentrated culture supernatants of $S$. flexneri strains were prepared as previously described $[12,16,21]$. Proteins were separated on SDS-PAGE stained with Coomassie blue 
or analyzed by Western blot using anti-IpaB and anti-IpaC monoclonal antibodies (mAbs) [22,23]. Virulence of the various strains was evaluated by testing bacterial ability to invade HeLa cells. The gentamicin protection assay, used to assess bacterial invasion of HeLa cells, was carried out as previously described $[23,24]$. Briefly, $2 \mathrm{~mL}$ of Shigella strains were grown to exponential growth phase $([\mathrm{OD} 600 \mathrm{~nm}]=0.4)$ and centrifuged onto plates containing $2 \times 10^{5} \mathrm{HeLa}$ cells at $2000 \times g$ for $10 \mathrm{~min}$. After $1 \mathrm{~h}$ of incubation at $37^{\circ} \mathrm{C}$, cells were washed three times with $2 \mathrm{~mL}$ EBSS-earle's balanced salt solution (Gibco, Belgium) and further incubated for $1 \mathrm{~h}$ in $2 \mathrm{~mL}$ MEM-minimum essential media (Gibco, Belgium) containing gentamicin at $50 \mu \mathrm{g} \mathrm{mL} \mathrm{L}^{-1}$ final concentration. The plates were washed three times and incubated for $15 \mathrm{~min}$ in Triton X-100 (0.1\% in PBS) to lyse the cells. Bacterial solutions were then diluted and plated on TSB-Agar containing appropriate antibiotics for colony-forming unit (CFU) counting.

\subsection{Lysozyme Sensitivity Assay and Polymorphonuclear Neutrophils (PMNs) Bacterial Infection}

The ability of Sfgtr4 mutant to resist lysosomal degradation was assessed as described previously [12]. Briefly, the pellet of bacteria grown to exponential phase was washed with Phosphate-Buffered Saline (PBS) (Gibco, Belgium) and then incubated for $4 \mathrm{~h}$ at $37^{\circ} \mathrm{C}$ with $40 \mu \mathrm{g} \mathrm{mL}{ }^{-1}$ of lysozyme and $0.1 \mathrm{mg} \mathrm{mL}^{-1}$ of lactoferrin (Sigma-Aldrich, Overijse, Belgium) before CFU counting. PMNs were isolated according to Pycock's method [25]. Bacterial infection by PMNs was performed as we described previously [12]. In brief, bacterial strains grown to exponential phase (optical density at $600 \mathrm{~nm}\left[\mathrm{OD}_{600 \mathrm{~nm}}\right]$ of 0.4 ) were diluted in PBS to obtain $10^{3}$ bacteria in $10 \mu \mathrm{L}$ and subsequently incubated with rabbit serum for $30 \mathrm{~min}$ at $37^{\circ} \mathrm{C}$. Next, $10^{5}$ PMNs put in Hank's Balanced Salt Solution (HBSS) (Gibco, Belgium) supplemented with $\mathrm{Mg}^{2+}, \mathrm{Ca}^{2+}$, and Gelatin $(0.1 \%)$ was added to the bacterial solution and incubated during $45 \mathrm{~min}$ at $37^{\circ} \mathrm{C}$. The reaction was stopped by incubating at $4{ }^{\circ} \mathrm{C}$ for $10 \mathrm{~min}$ at and PMNs were lysed by adding $100 \mu \mathrm{L}$ of sterile water. Lastly, bacteria were plated on TSB-Agar and incubated overnight at $37^{\circ} \mathrm{C}$ before CFU counting.

\subsection{Measuring the Biofilm Formation by Shigella}

Two methods were used to monitor whether Shigella forms biofilm. First, we used the classical Crystal Violet's (CV) binding assay [26]. Briefly, overnight cultures of bacteria were seeded in polystyrene 24-wells patches (Costar $\left.{ }^{\circledR}\right)$ (Fisher scientific, Belgium) at $10^{7} \mathrm{CFU} / \mathrm{mL}$ in TSB medium supplemented with adequate antibiotics as needed. Bacteria were brooded for $24 \mathrm{~h}$ at $37^{\circ} \mathrm{C}$. Floating bacteria were removed by washing. The bacterial biofilm forming in the air-liquid interface was colored with $0.5 \%$ CV's solution for 20 min before washing with distilled water. Finally, the biofilm was fixed with $1 \mathrm{~mL}$ of absolute ethanol and was unstuck by means of a Pasteur pipette. The $\left[\mathrm{OD}_{570 \mathrm{~nm}}\right]$ was measured and the results were expressed in percentage of the membership regarding wild-type strain.

The second method used here is based on the Biofilm Ring Test ${ }^{\circledR}$ (BioFilm control, France) which is more sensitive and consisted of the immobilization of magnetic beads by attached cells as previously described [27]. Consequently, the more beads are entrapped by cells, the less they are detectable after magnetization, and subsequently, the more cells are attached to the surface. The assay was carried out in modified polystyrene 96-well microtiter plates as described in [27]. Wells containing Brain Heart Infusion (BHI) medium were inoculated with a bacterial suspension and magnetic beads and were incubated at $37^{\circ} \mathrm{C}$ without shaking. Wells were scanned, magnetized, and re-scanned. Free beads were attracted to the center of the bottom of wells, forming a black spot, while beads blocked by the biofilm remained in place. As a control, BHI medium showed a centered spot. Images of each well before $\left(\mathrm{I}_{0}\right)$ and after $\left(\mathrm{I}_{1}\right)$ magnetization were compared with the Biofilms Control ${ }^{\circledR}$ software that gives a Biofilm Index (BFI). A value of BFI $>7$ indicates a high mobility of beads under magnet action (i.e., control wells) (Biofilm control, France). BFI $<2$ represents a full immobilization of beads (positive biofilm), while a BFI values ranging between 2 and 7 corresponded to the course of biofilm formation. Optimal condition leading to biofilm formation was determined by growing bacteria from $1 \mathrm{~h}$ to $6 \mathrm{~h}$. 


\subsection{Protein Purification and Maltose Binding Protein (MBP) Pull-Down Assay}

Plasmids pKA20 (MBP-SfPgdA), pKA22 (SfPgdA-His), and pKA23 (SfGtr4-His) were constructed by cloning PCR DNA fragments (856-bp BamHI-PstI, 1102-bp NcoI-BglII, and 855-bp NcoI-BglII) into the corresponding sites of pMALCRI or pQE60 vectors (Qiagen, France). To produce recombinants MBP-SfPgdA, SfPgdA-His, and SfGtr4-His proteins; overnight culture of E. coli carrying pKA20, pKA22, or pKA23 was subcultured into $100 \mathrm{~mL}$ of L-broth. The cultures were grown with vigorous shaking at $37^{\circ} \mathrm{C}$ until an $\left[\mathrm{OD}_{600 \mathrm{~nm}}\right]$ of 0.8 was reached. Next, isopropyl $\beta$-D-1-thiogalactopyranoside (IPTG) was added at a final concentration of $0.1 \mathrm{mM}$, and growth was continued for an additional $3 \mathrm{~h}$. Cells were harvested by centrifugation and stored at $-80^{\circ} \mathrm{C}$ until use. Cell pellets were thawed on ice, suspended in $1.5 \mathrm{~mL}$ of lysis buffer supplemented with Lysozyme, $1 \%$ Triton X-100 and protease inhibitor cocktail (Roche), broken by ultrasonic treatment, and then centrifuged at $6000 \mathrm{~g}$. Next, the supernatant was retained for further protein purification steps. The maltose-fused proteins MBP-SfPgdA bound to $100 \mu \mathrm{L}$ of amylose beads were mixed with a cleared extract of TOP10 producing recombinant SfPgdA-His or SfGtr4-His hybrid proteins and further incubated for $1 \mathrm{~h}$ at $4{ }^{\circ} \mathrm{C}$. Supernatants were removed by centrifugation, and beads were washed six times with washing buffer ( $\mathrm{pH}$ 7.4). After the final wash, supernatants were removed, and $100 \mu \mathrm{L}$ of $10 \mathrm{mM}$ of saccharose was added to elute MBP-fused protein. From each eluate, 10 to $20 \mu \mathrm{L}(\sim 100 \mathrm{ng})$ of proteins was separated by SDS-PAGE and further analyzed by Western blot using an anti-His mAb and anti-GST and anti-MBP polyclonal antibodies.

\subsection{Detection of the Glycosyltransferase Enzymatic Activity}

The Gtr enzymatic activity of MBP-SfGtr4 was monitored in 96-well microplates. In brief, we used an enzymatic chain reaction which allows the detection of the uridine diphosphate (UDP) product by coupling its formation to the nicotinamide adenine dinucleotide deshyrogenase (NADH) mediated reduction of pyruvate to lactate by lactic dehydrogenase. The pyruvate is produced from phospho(enol)pyruvate (PEP), which phosphorylates UDP in the presence of the pyruvate kinase. As the concentration of NADH decreased, the fluorescent signal at the emission wavelength of NADH is also decreased. The signal decrease was monitored at $\left[\mathrm{OD}_{460} \mathrm{~nm}\right]$ using an FLX 800 Microplate Fluorescence plate reader with excitation at $340 \mathrm{~nm}$ as previously described [28]. For the experimental procedure, each reaction contained buffer (50 $\mathrm{mM}$ 4-(2-hydroxyethyl)-1-piperazineethanesulfonic acid (HEPES), pH 7.9 (Clinisciences, France), $5 \mathrm{mM} \mathrm{MgCl}_{2}$ and, except where noted, $0.2 \%$ Triton (X-100), $0.5 \mathrm{mM}$ PEP, $0.2 \mathrm{U} / \mu \mathrm{L}$ of lactic dehydrogenase, $3 \mathrm{U} / \mu \mathrm{L}$ of pyruvate kinase, $0.25 \mathrm{mM} \mathrm{NADH}, 15 \%$ methanol, an appropriate amount of UDP-N-Acetylglucosamine (GlcNAc), lipid A from E. coli, and $0.5-10 \mu \mathrm{L}$ of the enzyme. The total volume of each reaction was $100 \mu \mathrm{L}$. All the components, except for the MBP-SfGtr4 substrates and MBP used as a negative control, were premixed in a reservoir and dispensed into each well. The substrates were then added to the reaction mixtures and incubated for 5-10 min until a stable background rate was achieved. MBP-SfGtr4 was then added, and the fluorescence was monitored after $20 \mathrm{~min}$.

\section{Results}

\subsection{Purified SfGtr4 Exhibits a Glycosyltransferase Enzymatic Activity}

The SfpgdA-Sfgtr4-virK-msbB2 genes, encoded by the virulence plasmid pWR100, were well conserved in Enteroaggregative Escherichia coli (EAEC) [1] (Figure 1A). Due to an in-frame mutation, sequence comparison revealed that the last 74 residues of SfGtr4 are missing from CapU (Figure 1B,C). The Sfgtr4 gene encodes a putative Gtr related to the GT-4 family. To investigate the enzymatic activity of SfGtr4, we adapted a previously described fluorescence assay (see Material and Methods; $M \& M)$. Briefly, we used an enzymatic chain reaction which facilitates the detection of UDP product by coupling its formation to the NADH-mediated reduction of pyruvate to lactate (Figure 2A). First, we constructed plasmid pKA20 producing MBP-SfGtr4. To monitor SfGtr4's potential glycosylation activity, $4 \mu \mathrm{g}$ of purified recombinant MBP-SfGtr4 protein was added to the reaction mixture. As shown 
in Figure 2B, a 50\% drop in fluorescence was detected, while no change was seen using purified MBP alone (Figure 2B). Our result demonstrates the role of SfGtr4 as a Glycosyltransferase mediating Glc transfer from UDP-Glc to lipid A (Figure 2B).

A/

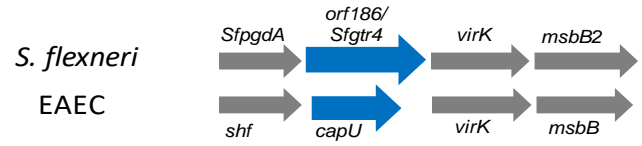

B/
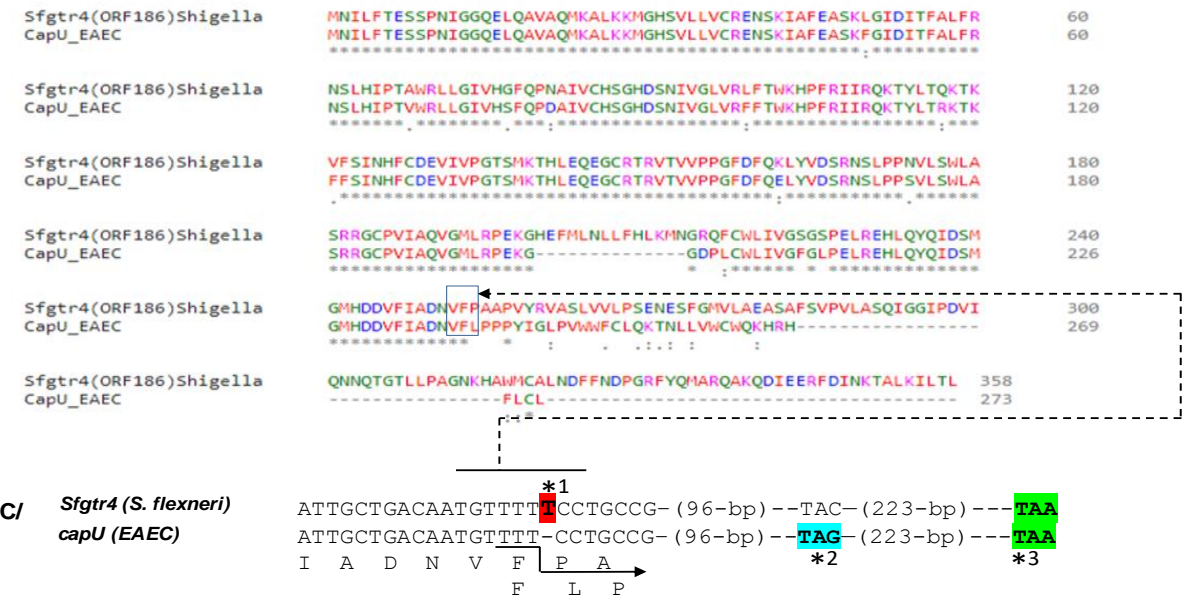

Figure 1. Conservation and sequence analysis between Shigella flexneri and Enteroaggregative E. coli (EAEA). (A) Conservation of the sfpgdA-sfgtr4-virK-msbB2 cluster between S. flexneri and Enteroaggregative Escherichia coli (EAEC). (B) Sequence alignment between Sfgtr4 and CapU proteins using Clastalow version 1.2.4 (C) Due to the absence of thymine (T) ( $\left.{ }^{*} 1\right)$ in cap $U$, the last 74 carboxyl residues of Sfgtr 4 are missing in CapU. Numbers 96 and 223 correspond to fully conserved nucleotides. Arrow points to the translational shift within CapU which is prematurely stopped at codon TAG ( $\left.{ }^{*} 2\right)$. If the thymine $\mathrm{T}\left({ }^{*} 1\right)$ was conserved in capU, both Sfgtr4 and capU would share the same stop codon (*3).

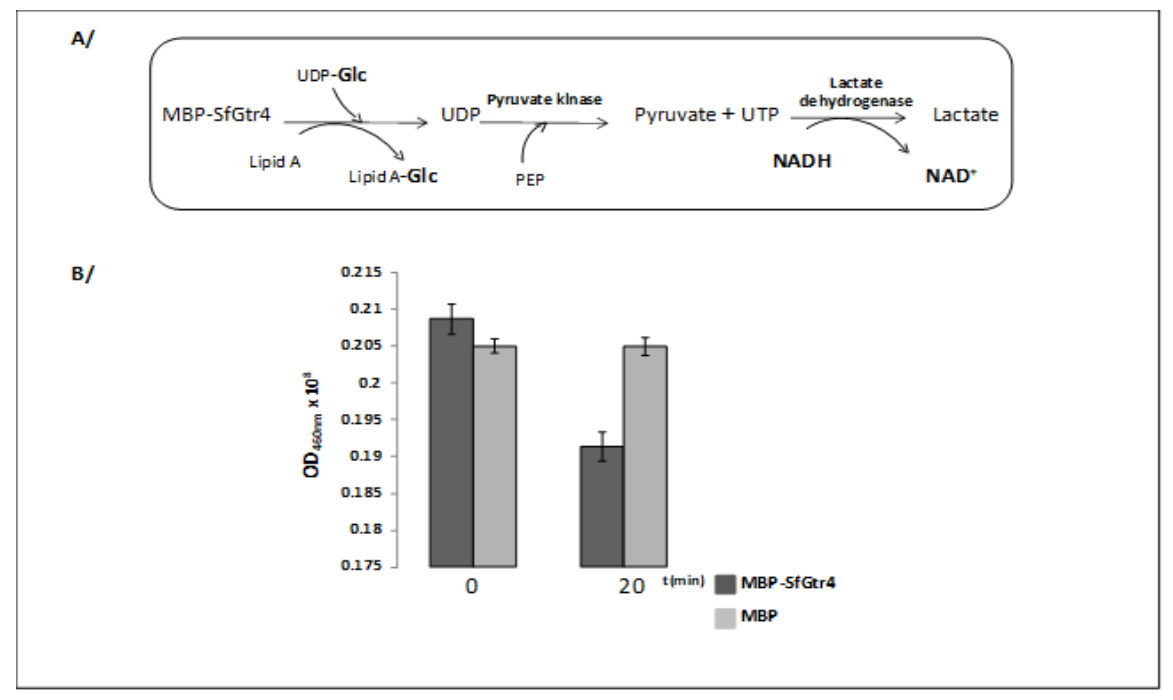

Figure 2. SfGtr4 exhibits a glycosyltransferase activity. (A) Schematic representation of the cascade of reaction used to monitor the enzymatic activity of maltose binding protein (MBP)-SfGtr4 protein. (B) The Gtr activity was monitored by measuring the decreased of NADH fluorescence 20 min post-incubation. Purified MBP was used as a negative control. This experiment was performed in triplicate. 
3.2. Inactivation of Sfgtr4 or SfpgdA Gene Reduces Shigella Entry into HeLa Cells Without Impairing Effectors Secretion via the T3SA

To investigate the role of SfGtr4 in virulence, we inactivated its corresponding gene on pWR100. The mutant was first tested in vitro for effectors secretion via the Mxi-Spa T3SA. Proteins of the supernatant, obtained either under constitutive or Congo red (CR) inducible secretion condition, were separated by SDS-PAGE stained with Coomassie blue or immunoblotted using anti-IpaB and IpaC mAbs. Both Sfgtr4 and SfpgdA mutants showed wild-type (WT) secretion profile (Figure 3A). As expected, no secretion was seen with the mxiD T3S secretion-deficient mutant, except for SepA and IcsA, the secretion of which is T3S-independent [19]. Thus, at least in vitro, both SfPgdA and SfGtr4 are not required for $\mathrm{T} 3$ secretion.

A/

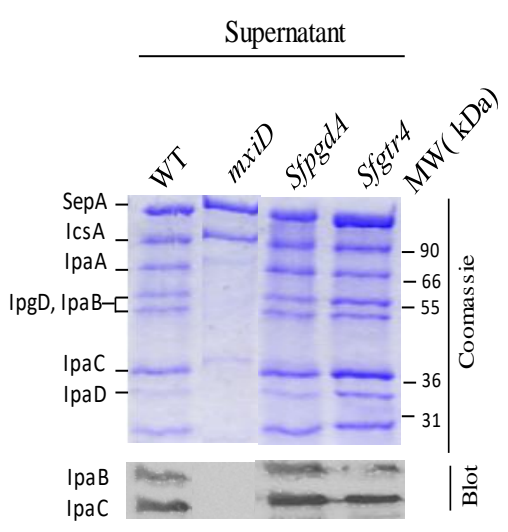

B/

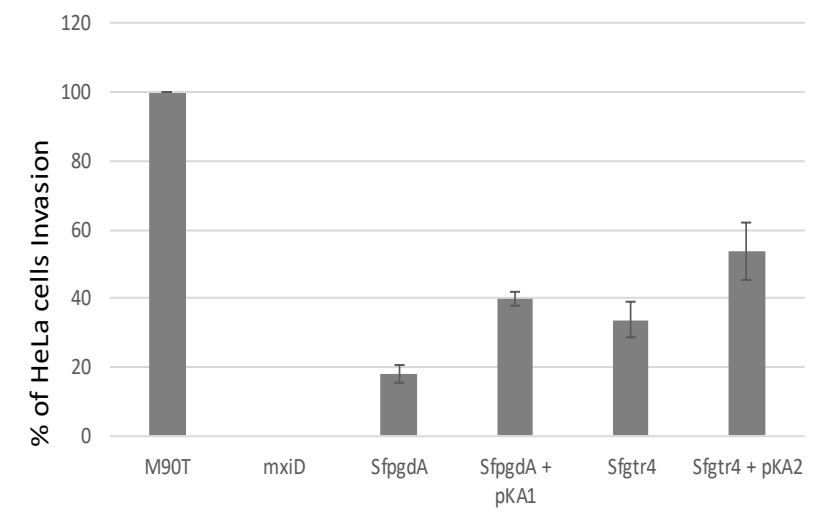

Figure 3. Inactivation of Sfgtr4 or SfpgdA impairs bacterial entry into HeLa cells without affecting secretion via T3SA. (A) Proteins in the supernatant were precipitated from wild-type strain (WT), SfpgdA, and Sfgtr 4 mutants and separated on SDS-PAGE stained with Coomassie blue or blotted using anti IpaB and IpaC mAbs. The mxiD mutant was used as a T3S secretion deficient control [19]. (B) Bacterial entry into HeLa cells was checked using the gentamicin protection assay [23]. Semi-confluent monolayer of HeLa cells was infected by WT, mutants SfpgdA and Sfgtr4, and their corresponding complemented strains with plasmid pKA1 (SfPgdA) or pKA2 (SfGtr4). Following bacterial entry, infected cells were incubated for $2 \mathrm{~h}$ at $37^{\circ} \mathrm{C}$ and then lysed by Triton X-100. Bacterial dilutions were further plated on agar-plates, incubated at $37^{\circ} \mathrm{C}$ for $16 \mathrm{~h}$ before $\mathrm{CFU}$ counting. Shown data are the mean of three independent experiments, and each one was performed in triplicate $(n=3)$.

Secretion of effectors via the T3SA is often coupled to the capacity of Shigella to invade cultured cells [2]. To assess whether inactivation of Sfgtr4 or SfpgdA affects bacterial entry into HeLa cells, we performed a gentamycin protection invasion assay. Compared to WT strain, the invasive capacity of the two Sfgtr4 and SfpgdA mutants was reduced by 3- and 4-fold, respectively. A complementation experiment using SfpgdA/pKA1 and Sfgtr4/pKA2 strains revealed $40 \%$ and 50\% WT invasion recovery, respectively (Figure 3B). This result is likely attributed to the unbalanced single SfPdgA or SfGtr4 overproduction in these strains. We conclude that both SfGtr4 and SfPgdA are prerequisite for efficient HeLa cells invasion by Shigella.

\subsection{Inactivation of Sfgtr4 Gene Enhances Sensitivity to Lysozyme Action and Increases Bacterial Killing by} Polymorphonuclear Neutrophils (PMNs)

We have previously shown that inactivation of $S f p d g A$ rendered Shigella more sensitive to lysozyme action [12]. A similar phenotype was obtained here with the Sfgtr4 mutant, roughly rescued upon complementation with plasmid pKA2 (SfGtr4) (Figure 4A). As a control, we used the SfpgdA mutant, 
which exhibited less pronounced sensitivity compared to the Sfgtr4 mutated strain (Figure 4A). Next, we tested whether simultaneous mutation of the two studied genes would strongly affect sensitivity to lysozyme action. For that, we constructed a Shigella strain lacking both SfpgdA and Sfgtr4 genes and found that the latter exhibited the Sfgtr4 mutant's sensitivity to lysozyme action (Figure 4A). The parental phenotype was partially restored ( 70\%) upon complementation with plasmid pKA85 producing both SfPgdA and SfGtr4 (Figure 4A). This result indicates the lack of a synergetic effect in the double mutant.

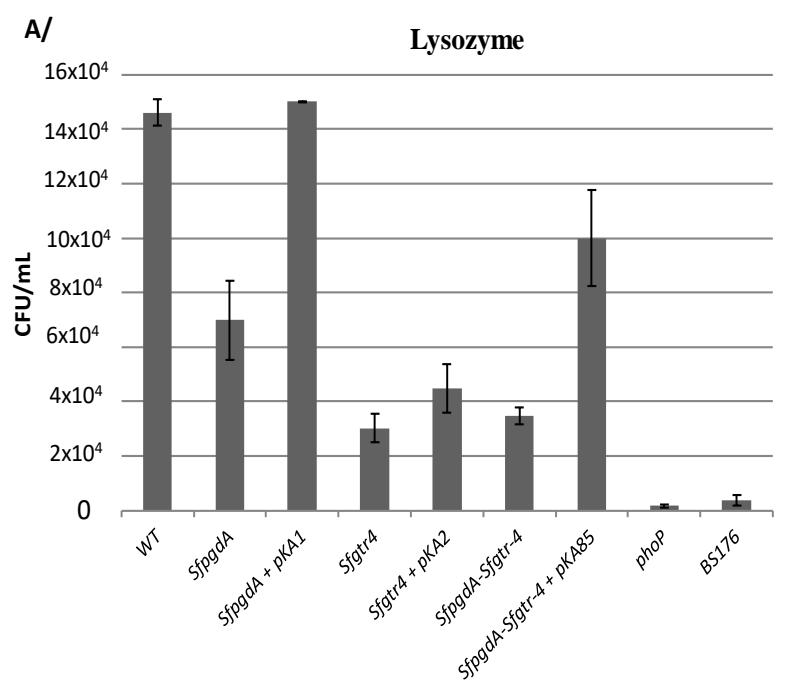

B/

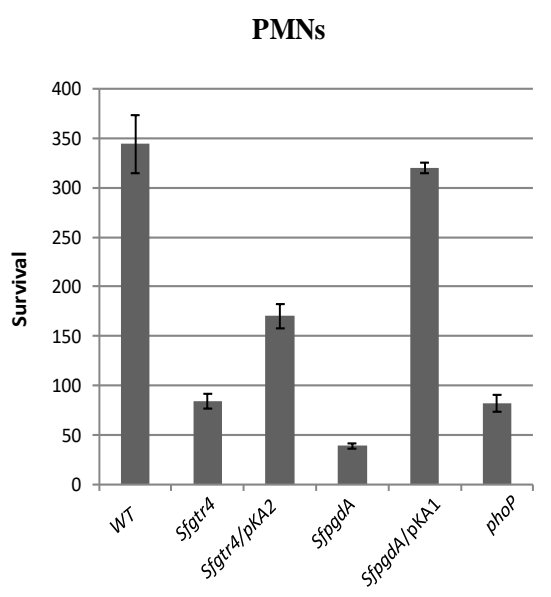

Figure 4. Inactivation of the Sfgtr4 gene increases sensitivity to lysozyme and enhances bacterial killing by polymorphonuclears neutrophils (PMNs). (A) Bacteria grown to exponential phase were incubated for $4 \mathrm{~h}$ at $37^{\circ} \mathrm{C}$ with lysozyme from chicken egg $\left(40 \mu \mathrm{g} \mathrm{mL}^{-1}\right)$ supplemented with lactoferrin $\left(0.1 \mu \mathrm{g} \mathrm{mL}{ }^{-1}\right) .10^{3}$ bacteria were incubated for $30 \mathrm{~min}$ with serum of rabbit at $37^{\circ} \mathrm{C}$ and further incubated for 45 min with PMNs extracted from human blood. (B) PMNs were lysed using distillated water before $\mathrm{CFU}$ counting after $24 \mathrm{~h}$ of incubation at $37^{\circ} \mathrm{C}$. Strains used are: WT, mutants: $S f p g d A$, Sfgtr4, SfpgdA-Sfgtr4 (double mutant), and phoP. Plasmids pKA1 (SfPgdA), pKA2 (SfGtr4), and pKA85 (SfPgdA and StGtr4) were used in the complementation experiments. Shown data are the mean of three independent experiments and each one was performed in triplicate $(n=3)$.

It is well established that among bactericidal mechanisms elaborated by PMNs, some require fusion of bacteria in phagosomes with granules that contain high concentrations of lysozyme and lactoferrin [24]. Thus, we investigated the behavior of the Sfgtr4 mutant upon PMNs infection. As for the SfpgdA and phoP mutants [12], used here as a control, inactivation of Sfgtr4 reduced bacterial persistence within PMNs, a phenotype that was partially rescued ( $50 \%)$ upon complementation with plasmid pKA2 (Figure 4B). In contrast, compared to the Sfgtr4 mutant, survival of the SfpgdA mutant was drastically affected, while strain $S f p g d A / p K A 1$ recovered almost the parental phenotype. SfGtr4 is therefore required for Shigella survival within PMNs.

\subsection{Inactivation of Sfgtr4 or SfpgdA Accelerates Biofilm Appearance}

Both SfGtr4 and SfPgdA are related to bacterial proteins required for biofilm formation, a process that remains poorly studied in Shigella. We first tested the capacity of S. flexneri strains to adhere on polystyrene plastic surfaces using the CV binding assay (see M\&M). Although WT Shigella does not efficiently adhere to this type of support compared to the strong adhesion seen with the Pseudomonas aeruginosa PAO1 strain, we found that WT strain, grown for $24 \mathrm{~h}$ at $37^{\circ} \mathrm{C}$, formed biofilm (Figure 5A). Comparatively, the Sfgtr4 mutant exhibited an increased bacterial adhesion $(\sim 80 \%)$, a phenotype that was almost restored to the WT level in strain Sfgtr4/pKA2 (Figure 5A). In contrast, only a limited increase in adhesion ( $17 \%$ ) was seen in the SfpgdA mutant (Figure 5A). All observed phenotypes were 
not associated with differential bacterial growth since the growth rate of either WT, SfpgdA, or Sfgtr4 mutant was equivalent (data not shown). Compared to WT Shigella, Pseudomonas aeruginosa (PAO1) strain exhibited 2.4-fold more abundant biofilm (data not shown).
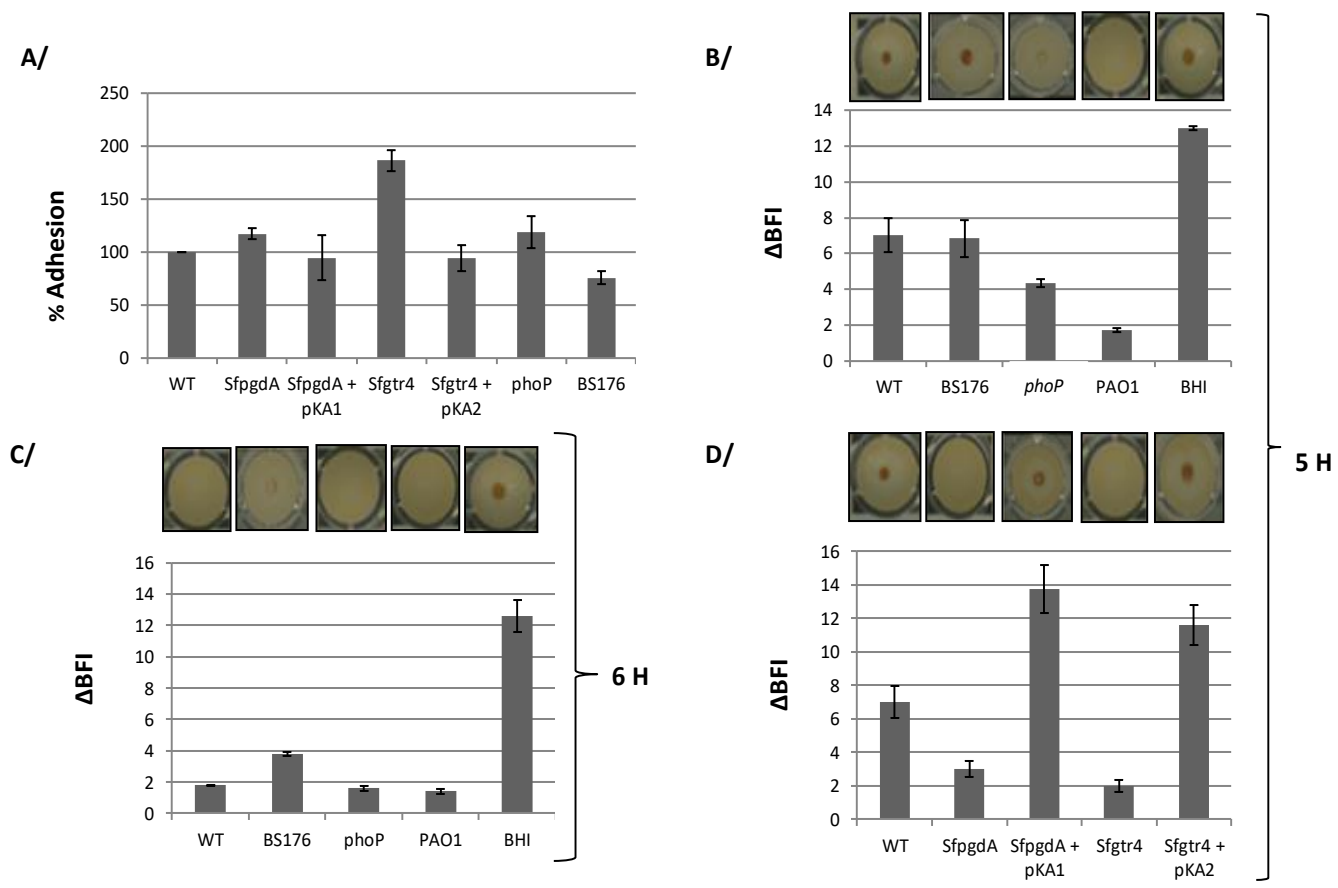

Figure 5. S. flexneri forms biofilm and deletion of either SfpgdA or Sfgtr4 accelerates this formation. (A) Quantification of biofilm formation by crystal violet $(\mathrm{CV})$ assay. Bacteria were grown in tryptic casein soy broth (TSB) medium during $24 \mathrm{~h}$ at $37^{\circ} \mathrm{C}$ and Biofilm formation was monitored by measuring the absorbance at $570 \mathrm{~nm}$. Strains used are WT, mutants SfpgdA, Sfgtr4, phoP, and BS176 (pWR100 cured S. flexneri strain). Biofilm formation was also measured using the biofilm ring test after bacterial incubation at $37^{\circ} \mathrm{C}$ during $5 \mathrm{~h}(\mathbf{B}, \mathbf{D})$ and $6 \mathrm{~h}(\mathrm{C}) . \triangle \mathrm{BFI}$ (Biofilm Formation Index) represents (BFI controls-BFI samples). Plasmids pKA1(SfPgdA) and pKA2 (SfGtr4) were used in the complementation experiments. Wild-type P. aeruginosa (PAO1) and BHI medium were used as positive and negative controls, respectively. Shown data are the mean of three independent experiments, and each one was performed in triplicate $(n=3)$.

To gain further evidence in support of the biofilm formation, we next use the ring sensitive assay (M\&M). Wild-type Shigella was grown at $37^{\circ} \mathrm{C}$ under various incubation times to determine condition during which a centered spot at the bottom of the well would disappear (M\&M). Up to 5 h of growth, the spot was still detectible (Figure 5B). Interestingly, after $6 \mathrm{~h}$ growth, no spot was seen, suggesting biofilm formation by WT Shigella was completed (Figure 5C). As a control, biofilm formation in strain PAO1 occurred within $2 \mathrm{~h}$ of growth (Figure 5B,C). As a confirmation these beads were not blocked by other factors other than sessile cells, BHI medium showed a centered spot (Figure 5B,C).

Having optimized the condition under which WT Shigella developed biofilm, we next explored the behavior of Sfgtr4 and SfpgdA mutants in this assay. Remarkably, no spot was seen with these strains after $5 \mathrm{~h}$ growth (Figure 5D). Interestingly, following $5 \mathrm{~h}$ growth, strains Sfgtr4/pKA2 and SfpgdA/pKA1 recovered the parental phenotype (Figure 5D).

\subsection{The phoP Mutant, but not the pWR100 Shigella Cured Strain, Increases Biofilm Formation}

PhoP regulates the transcription of SfpgdA-Sfgtr4-virK-msbB2 cluster, and SfPgdA protein is not produced by the phoP mutant [12]. Based on this, we investigated biofilm formation by the phoP mutant. In the CV binding assay, compared to WT strain, a relative increase in adhesion $(\sim 18 \%)$ was detected 
with the $p h o P$ mutant (Figure 5A). Furthermore, using the ring test, after $5 \mathrm{~h}$ growth, biofilm was seen with the phoP mutant, in a comparable manner to the SfpgdA or Sfgtr4 one (Figure 5B).

As the two studied genes are encoded by pWR100, we next carried out similar experiments using BS176, a pWR100 cured Shigella strain. In the CV binding assay, BS176 exhibited a $25 \%$ decreased adhesion compared to WT strain (Figure 5A). In the ring test, post $5 \mathrm{~h}$ growth, a spot was observed in the center of the well (Figure 5B). When tested after $6 \mathrm{~h}$ growth, the intensity of the spot was slightly reduced (Figure 5C). This result is likely to be associated with the fast growth of BS176 compared to WT strain (data not shown). We conclude that BS176 does not form biofilm, which strongly suggests the need for additional pWR100 encoding factors.

\subsection{SfGtr4 Interacts with SfPgdA}

The contiguous localization of SfpgdA and Sfgtr4 genes was associated with similar phenotype shared by their respective mutants: (i) reduced entry into HeLa cells; (ii) increased biofilm formation; and (iii) increased susceptibility to lysosomal degradation and killing by PMN. Such outcomes prompted us to investigate the potential interaction between their products. We therefore constructed two plasmids producing SfPgdA-His and SfGtr4-His and used another available one producing maltose binding protein fused to SfPgdA (MBP-SfPgdA) [12] (Table 1). Using the MBP pull-down assay (M\&M), we demonstrated that SfPgdA self-associates and interacts with SfGtr4 (Figure 6). As a control, no interaction was detected with MBP alone.
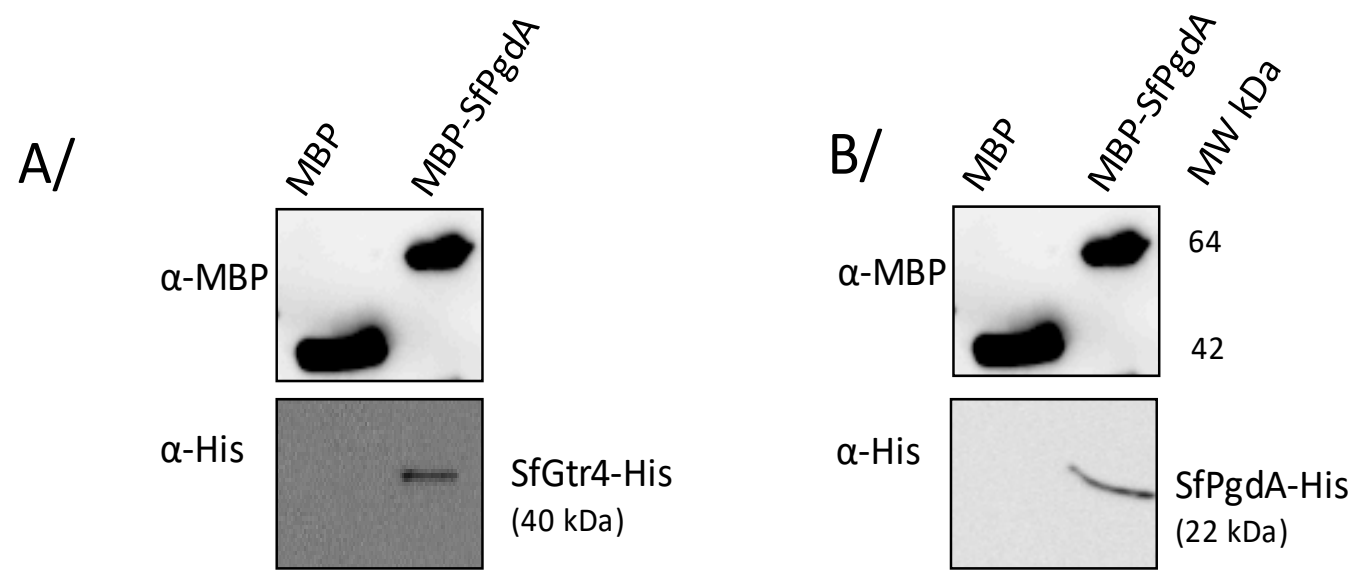

Figure 6. SfPgdA interacts with itself and with SfGtr4 using maltose binding protein (MBP) pull-down assay. MBP-SfPgdA bound to amylose beads was mixed with a cleared extract of $E$. coli strain producing either (A) recombinant His-SfGtr4 or (B) His-SfPgdA hybrid proteins. Saccharose was used to elute MBP-fused proteins. Aliquots of each eluted fraction were analyzed by Western blot using an anti-His $\mathrm{mAb}$ or anti-MBP polyclonal antibodies. Purified MBP alone was used as a negative binding control. MW: proteins molecular weight.

\section{Discussion}

The present study highlights the ability of bacterium of the genus Shigella to form biofilm. We report here that the deletion of either Sfgtr4 or SfpgdA genes accelerates biofilm appearance. Both SfGtr4 and SfPgdA are closely related to Shf and CapU proteins required for Enteroaggregative E. coli (EAEC) biofilm formation $[3,5,15]$. In the latter, strong aggregation in culture medium causes the spontaneous settling of cells onto the substratum and subsequently contributes to thick biofilm formation [3]. It was suggested that shf gene is not related to aggregation in the liquid phase and adhesion in the early phase but is required to form the multiple layers of biofilm in the maturation phase $[3,26]$. In addition, the same authors showed that strain mutated in capU gene exhibited parental EAEC biofilm, while our inactivation of the Sfgtr4 gene rather accelerated its appearance in Shigella. The difference between our data and those of Fujiyama et al. $[3,26]$ in EAEC could be due to the lack, within CapU, of the 
74 carboxyl-terminal residues of SfGtr4 (Figure 1B). Thus, production of SfGtr4 by WT Shigella prevents biofilm appearance, while its deletion rather mimics both WT EAEC and capU mutant phenotype.

Biofilm formation comprises different steps; one of the earliest is the attachment of the bacteria on the substrate. The absence of a biofilm formation in BS176 supports the existence of factors that are encoded by the pWR100 plasmid that are paramount in biofilm formation. In support of this, we demonstrated a molecular interaction between SfPgdA and SfGtr4. We have previously demonstrated that SfPgdA is associated to the bacterial membranes fraction [12]. It is likely that both proteins associate in a complex dealing with bacterial surface modifications. Comparatively, PGA production and excretion changes at the bacterial envelope were suggested to be associated with the PgaABCD complex of E. coli [5]. Biofilm is generally embedded in extracellular polysaccharide composed of PGA [29], and its formation is mediated by exopolysaccharide (EPS) production [30].

SfPgdA exhibits a peptidoglycan deacetylase (PGD) activity used by WT Shigella to resist lysozome hydrolase activity [12]. Considering that PG breaks, generated by PG hydrolase and facilitate biofilm formation [31], Shigella strain lacking SfPgdA becomes more sensitive to hydrolase, which in turn accelerates biofilm formation. Evidence supporting this finding was reported in Lactococcus lactis whose biofilm formation was mainly attributed to PG hydrolysis [30]. Moreover, PGA deacetylation in Streptococcus epidermidis contributes not only to biofilm development but also to immune evasion and virulence [18], a result that corroborates the recent role ascribed to SfPgdA in Shigella persistence within PMNs [12].

The virulence processes of pathogenic bacteria are frequently coupled to the secretion of effector molecules into the environment. T3SA is required for protein secretion that contributes to the virulence of several animal and plant pathogens $[2,32,33]$ and is responsible for Shigella entry into epithelial cells $[8,18]$. One unexpected result, obtained here, points to the reduced invasion of HeLa cells by SfpgdA and Sfgtr4 mutants, although both strains exhibit a functional T3SA. A similar phenotype was previously reported with the gtrV gene mutant of Shigella [34]. Interestingly, GtrV is involved in LPS glucosylation [34]. The invasion defect of the $g \operatorname{tr} V$ mutant was attributed to the increased length of the unglugosylated LPS, which consequently extend both the glucosylated one and the T3S needle $50 \mathrm{~nm}$ length entities [34]. One may consider that the lack of the Gtr enzymatic activity in the Sfgtr4 mutant could disrupt needle exposition at the bacterial surface, which consequently reduces the capacity to invade HeLa cells. Our invasion data revealed partial complementation using either sfgtr4/pKA2 or sfpgdA/pKA1 strains. Both pKA1 and pKA2 plasmids are derivative of the high copy number pUC18 vector known to constitutively overproduces proteins. As SfPgdA is associated with the bacterial membrane fraction [12] and interacts with SfGtr4, their single overproduction could significantly affect their function. Interestingly, in the lysozyme assay, simultaneous overproduction of both SfPgdA and SfGtr4, in the double SfpdgA-Sfgtr4 mutant, recovered almost 70\% of the WT phenotype. A finding that contrasts with the lowest complementation seen following individual overproduction of either SfpgdA or Sfgtr4. Such a finding suggests that the identified molecular interaction between SfPgdA and SfGtr4 is likely involved in the regulation of their functions.

How does Sfgtr4 mutant affect Shigella's resistance to lysozyme action? It is well documented that LPS glycosylation destabilizes the bacterial membrane, which facilitates lysozyme access to the PG layer [7]. For example, inactivation of Salmonella enterica ugtl gene, involved in heptose incorporation into lipid A, increased sensitivity to polymyxin B [18]. Interestingly, the SfpgdA-msbB2 locus and ugtl gene are both regulated by the PhoP/PhoQ regulatory system $[7,10]$. Since PhoP is involved in the regulation of genes remodeling bacterial cell envelope in response to changing environmental conditions, the increased biofilm formation detected in the $p_{o} P$ mutant is likely to be attributable at least to the lack of SfPgdA [12].

Based on our reconstituted in vitro Gtr enzymatic assay, we cannot precisely statute about Sfgtr4's target-either LPS or PG, or both? By transferring glucose to lipid I, SfGtr4 may glycosylate PG. Comparatively, MurG, a Gtr from E. coli, transfers a molecule of glucose to lipid I, which in turn 
generates lipid II, the PG precursor [28]. The lack of PG glycosylation in the Sfgtr4 mutant would amplify lysozyme action as previously reported for the namH mutant of Mycobacterium smegmatis [35].

To date, no investigation has identified the environmental ecology niches of Shigella yet. Here, we bring an additional contribution on biofilm formation by WT Shigella, a process that we showed here to be controlled by SfPgdA and SfGtr4 proteins. A previous study reported that exposition of WT Shigella flexneri to bile salts induced biofilm formation and revealed the role of LPS O-antigen synthesis in this process [36]. Indeed, various rough mutants or mutations in the gall gene, which catalyzes the formation of UDP-glucose, required for O-antigen synthesis, affect bile salts resistance. Another study reported that a Shigella strain lacking the ics $A$ gene, required for both adherence to cells and bacterial actin-based motility, abolished bile salt-induced biofilm formation [37]. Here, we showed that production and secretion of IcsA were not affected in the SfpgdA and Sfgtr4 mutants (Figure 3A), suggesting the existence of additional mechanisms involved in biofilm formation by Shigella. More recent study reported that the biofilm formation by Gram-negative Haemophilus influenzae was increased by deletion of galE gene and the remarkable availability of UDP-GlcNAc precursors [38]. The same study reported that the suppression of alternative lipooligosaccharides glycosyltransferase activity by UDP-Galactose epimerase enhanced murine lung infection and evasion of serum IgM [38].

The arising question here is why and when Shigella would need to form biofilm. If we consider that only 10 to 100 bacteria are sufficient to cause disease in humans, such bacterial dose cannot bind to a biofilm. Nevertheless, one could suggest that biofilm development is a transitory regulated event that occurres upon food contamination to help Shigella survival outside of its preferred habitat. This phenomenon may happen under environmental conditions during which the expression of a limited number of genes, including SfpgdA and Sfpgtr4, is downregulated.

Compared to macrophages, neutrophils prevent the escape of Shigella from phagocytic vacuoles in which the bacteria are killed [39]. The Sfgtr4 and SfpgdA mutants are both unable to escape PMNs killing. One might consider that the ability of WT Shigella to modify its surface, by targeting either PG and/or LPS, is to subvert bacterial detection by the host immune system. Neutrophil-phagocytosed Shigella are killed rapidly by different mechanisms including proteolytic enzymes, antimicrobial proteins, and reactive oxygen species [38]. Thus, further investigations are needed to unravel mechanisms underpinning Shigella's circumvention and/or counteraction of the host's immune responses.

Author Contributions: Conceptualization, L.B. and A.A.; Funding acquisition, A.A.; Investigation, A.K.-R. and L.B.; Methodology, A.K.-R.; Project administration, A.A.; Supervision, L.B. and A.A.; Validation, A.K.-R. and A.A.; Writing —original draft, A.A.; Writing-review \& editing, L.B. and A.A. All authors have read and agreed to the published version of the manuscript.

Funding: This work was supported by grants from the Belgian FRSM (Fonds National de la Recherche Scientifique Médicale: conventions: 3.4.623.06 and 3.4556.11). KRA was a recipient of a PhD fellowship from the Belgian Fonds National de Recherches Industrielles et Agronomiques (FRIA). A part of this work was supported by the welcome grant of the UM6P university of Benguérir, Morocco.

Acknowledgments: We thank Thierry Jouenne and Camille Macé for initiating biofilm assays and Hassan Rahmoune and Kamal El Bissati for critical reading of the manuscript.

Conflicts of Interest: The authors declare no conflict of interest.

\section{References}

1. Buchrieser, C.; Glaser, P.; Rusniok, C.; Nedjari, H.; D’Hauteville, H.; Kunst, F.; Sansonetti, P.; Parsot, C. The virulence plasmid pWR100 and the repertoire of proteins secreted by the type III secretion apparatus of Shigella flexneri. Mol. Microbiol. 2000, 38, 760-771. [CrossRef] [PubMed]

2. Parsot, C. Shigella type III secretion effectors: How, where, when, for what purposes? Curr. Opin. Microbiol. 2009, 12, 110-116. [CrossRef] [PubMed]

3. Fujiyama, R.; Nishi, J.; Imuta, N.; Tokuda, K.; Manago, K.; Kawano, Y. The shf Gene of a Shigella flexneri homologue on the virulent plasmid pAA2 of enteroaggregative escherichia coli 042 Is required for firm biofilm formation. Curr. Microbiol. 2008, 56, 474-480. [CrossRef] [PubMed] 
4. Forman, S.; Bobrov, A.G.; Kirillina, O.; Craig, S.K.; Abney, J.; Fetherston, J.D.; Perry, R.D. Identification of critical amino acid residues in the plague biofilm Hms proteins. Microbiology 2006, 152, 3399-3410. [CrossRef] [PubMed]

5. Itoh, Y.; Rice, J.D.; Goller, C.; Pannuri, A.; Taylor, J.; Meisner, J.; Beveridge, T.J.; Preston, J.F.; Romeo, T. Roles of pgaABCD Genes in synthesis, modification, and export of the escherichia coli biofilm adhesin

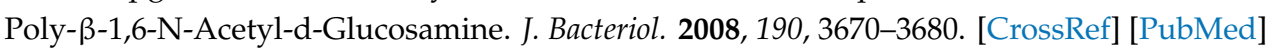

6. Perry, R.D.; Bobrov, A.G.; Kirillina, O.; Jones, H.A.; Pedersen, L.; Abney, J.; Fetherston, J.D. Temperature regulation of the Hemin storage (Hms+) phenotype of Yersinia pestis is posttranscriptional. J. Bacteriol. 2004, 186, 1638-1647. [CrossRef] [PubMed]

7. Shi, Y.; Cromie, M.J.; Hsu, F.-F.; Turk, J.; Groisman, E.A. PhoP-regulated Salmonella resistance to the antimicrobial peptides magainin 2 and polymyxin B. Mol. Microbiol. 2004, 53, 229-241. [CrossRef]

8. Vuong, C.; Kocianova, S.; Voyich, J.M.; Yao, Y.; Fischer, E.R.; DeLeo, F.R.; Otto, M. A Crucial role for exopolysaccharide modification in bacterial biofilm formation, immune evasion, and virulence. J. Boil. Chem. 2004, 279, 54881-54886. [CrossRef]

9. O’Toole, G.A.; Kaplan, H.B.; Kolter, R. Biofilm formation as microbial development. Annu. Rev. Microbiol. 2000, 54, 49-79. [CrossRef]

10. Goldman, S.R.; Tu, Y.; Goldberg, M.B. Differential regulation by magnesium of the two MsbB paralogs of Shigella flexneri. J. Bacteriol. 2008, 190, 3526-3537. [CrossRef]

11. Lin, Z.; Cai, X.; Chen, M.; Ye, L.; Wu, Y.; Wang, X.; Lv, Z.; Shang, Y.; Qu, D. Virulence and stress responses of Shigella flexneri regulated by PhoP/PhoQ. Front. Microbiol. 2018, 8, 2689. [CrossRef] [PubMed]

12. Kaoukab-Raji, A.; Biskri, L.; Bernardini, M.; Allaoui, A. Characterization of SfPgdA, a Shigella flexneri peptidoglycan deacetylase required for bacterial persistence within polymorphonuclear neutrophils. Microbes Infect. 2012, 14, 619-627. [CrossRef] [PubMed]

13. Makino, K.; Ishii, K.; Yasunaga, T.; Hattori, M.; Yokoyama, K.; Yutsudo, C.H.; Kubota, Y.; Yamaichi, Y.; Iida, T.; Yamamoto, K.; et al. Complete nucleotide sequences of $93-\mathrm{kb}$ and 3.3-kb plasmids of an enterohemorrhagic Escherichia coli O157:H7 derived from Sakai outbreak. Curr. Neuropharmacol. 1998, 5, 1-9. [CrossRef] [PubMed]

14. Yethon, J.A.; Vinogradov, E.; Perry, M.B.; Whitfield, C. Mutation of the lipopolysaccharide core glycosyltransferase encoded by waaG destabilizes the outer membrane of escherichia coli by interfering with core phosphorylation. J. Bacteriol. 2000, 182, 5620-5623. [CrossRef] [PubMed]

15. Lairson, L.L.; Henrissat, B.; Davies, G.J.; Withers, S. Glycosyltransferases: Structures, Functions, and Mechanisms. Annu. Rev. Biochem. 2008, 77, 521-555. [CrossRef] [PubMed]

16. Allaoui, A.; Sansonetti, P.J.; Parsot, C. MxiJ, a lipoprotein involved in secretion of Shigella Ipa invasins, is homologous to YscJ, a secretion factor of the Yersinia Yop proteins. J. Bacteriol. 1992, 174, 7661-7669. [CrossRef]

17. Demarre, G.; Guerout, A.-M.; Matsumoto-Mashimo, C.; Rowe-Magnus, D.; Marlière, P.; Mazel, D. A new family of mobilizable suicide plasmids based on broad host range R388 plasmid (IncW) and RP4 plasmid $(\operatorname{IncP} \alpha)$ conjugative machineries and their cognate Escherichia coli host strains. Res. Microbiol. 2005, 156, 245-255. [CrossRef]

18. Sansonetti, P.J.; Ryter, A.; Clerc, P.; Maurelli, A.T.; Mounier, J. Multiplication of Shigella flexneri within HeLa cells: Lysis of the phagocytic vacuole and plasmid-mediated contact hemolysis. Infect. Immun. 1986, 51, 461-469. [CrossRef]

19. Allaoui, A.; Sansonetti, P.J.; Parsot, C. MxiD, an outer membrane protein necessary for the secretion of the Shigella flexneri Ipa invasins. Mol. Microbiol. 1993, 7, 59-68. [CrossRef]

20. Ménard, R.; Sansonetti, P.J.; Parsot, C. Nonpolar mutagenesis of the ipa genes defines IpaB, IpaC, and IpaD as effectors of Shigella flexneri entry into epithelial cells. J. Bacteriol. 1993, 175, 5899-5906. [CrossRef] [PubMed]

21. Magdalena, J.; Hachani, A.; Chamekh, M.; Jouihri, N.; Gounon, P.; Blocker, A.; Allaoui, A. Spa32 regulates a switch in substrate specificity of the Type III secreton of Shigella flexneri from needle components to Ipa Proteins. J. Bacteriol. 2002, 184, 3433-3441. [CrossRef] [PubMed]

22. Barzu, S.; Nato, F.; Rouyre, S.; Mazie, J.C.; Sansonetti, P.; Phalipon, A. Characterization of B-cell epitopes on IpaB, an invasion-associated antigen of Shigella flexneri: Identification of an immunodominant domain recognized during natural infection. Infect. Immun. 1993, 61, 3825-3831. [CrossRef] [PubMed] 
23. Phalipon, A.; Arondel, J.; Nato, F.; Rouyre, S.; Mazie, J.C.; Sansonetti, P.J. Identification and characterization of B-cell epitopes of IpaC, an invasion-associated protein of Shigella flexneri. Infect. Immun. 1992, 60, 1919-1926. [CrossRef] [PubMed]

24. Roy, S.; Singh, V.; More, T. Electrophoretic detection of myeloperoxidase, protease, lactoferrin and lysozyme in buffalo polymorphonuclear granular acid extracts. Veter- Res. Commun. 1997, 21, 325-334. [CrossRef] [PubMed]

25. Pycock, J.; Allen, W.E.; Morris, T. Rapid, single-step isolation of equine neutrophils on a discontinuous Percoll density gradient. Res. Veter-Sci. 1987, 42, 411-412. [CrossRef]

26. Peeters, E.; Nelis, H.J.; Coenye, T. Comparison of multiple methods for quantification of microbial biofilms grown in microtiter plates. J. Microbiol. Methods 2008, 72, 157-165. [CrossRef]

27. Chavant, P.; Gaillard-Martinie, B.; Talon, R.; Hebraud, M.; Bernardi, T. A new device for rapid evaluation of biofilm formation potential by bacteria. J. Microbiol. Methods 2007, 68, 605-612. [CrossRef]

28. Chen, L.; Men, H.; Ha, S.; Ye, X.-Y.; Brunner, L.; Hu, Y.; Walker, S. Intrinsic Lipid Preferences and Kinetic Mechanism ofEscherichia coliMurGt. Biochemistry 2002, 41, 6824-6833. [CrossRef]

29. Maira-Litrán, T.; Kropec, A.; Abeygunawardana, C.; Joyce, J.; Mark, G.; Goldmann, D.A.; Pier, G.; Iii, G.M. Immunochemical properties of the staphylococcal Poly-N-Acetylglucosamine surface polysaccharide. Infect. Immun. 2002, 70, 4433-4440. [CrossRef]

30. Cramton, S.E.; Gerke, C.; Schnell, N.F.; Nichols, W.W.; Götz, F. The Intercellular Adhesion (ica) Locus Is present in staphylococcus aureus and is required for biofilm formation. Infect. Immun. 1999, 67, 5427-5433. [CrossRef]

31. Mercier, C.; Durrieu, C.; Briandet, R.; Domakova, E.; Tremblay, J.; Buist, G.; Kulakauskas, S. Positive role of peptidoglycan breaks in lactococcal biofilm formation. Mol. Microbiol. 2002, 46, 235-243. [CrossRef] [PubMed]

32. Cornelis, G.R. The type III secretion injectisome. Nat. Rev. Genet. 2006, 4, 811-825. [CrossRef] [PubMed]

33. Hueck, C.J. Type III protein secretion systems in bacterial pathogens of animals and plants. Microbiol. Mol. Boil. Rev. 1998, 62, 379-433. [CrossRef]

34. West, N.P.; Sansonetti, P.; Mounier, J.; Exley, R.M.; Parsot, C.; Guadagnini, S.; Prévost, M.-C.; Prochnicka-Chalufour, A.; Delepierre, M.; Tanguy, M.; et al. Optimization of virulence functions through glucosylation of Shigella LPS. Science 2005, 307, 1313-1317. [CrossRef] [PubMed]

35. Raymond, J.B.; Mahapatra, S.; Crick, D.C.; Pavelka, M.S. Identification of thenamHGene, encoding the hydroxylase responsible for the N-Glycolylation of the mycobacterial peptidoglycan. J. Boil. Chem. 2004, 280, 326-333. [CrossRef]

36. Nickerson, K.P.; Chanin, R.B.; Sistrunk, J.R.; Rasko, D.A.; Fink, P.J.; Barry, E.M.; Nataro, J.P.; Faherty, C.S. Analysis of Shigella flexneri resistance, biofilm formation, and transcriptional profile in response to bile salts. Infect. Immun. 2017, 85, e01067:1-e01067:16. [CrossRef]

37. Köseoğlu, V.K.; Hall, C.P.; Rodríguez-López, E.M.; Agaisse, H. The autotransporter IcsA promotes Shigella flexneri biofilm formation in the presence of bile salts. Infect. Immun. 2019, 87, e00861-e008618. [CrossRef]

38. Wong, S.M.; Jackson, M.D.; Akerley, B.J. Suppression of alternative lipooligosaccharide Glycosyltransferase activity by UDP-Galactose epimerase enhances murine lung infection and evasion of serum $\operatorname{IgM}$. Front. Microbiol. 2019, 9, 160. [CrossRef]

39. Weinrauch, Y.; Drujan, D.; Shapiro, S.D.; Weiss, J.; Zychlinsky, A. Neutrophil elastase targets virulence factors of enterobacteria. Nature 2002, 417, 91. [CrossRef]

(C) 2020 by the authors. Licensee MDPI, Basel, Switzerland. This article is an open access article distributed under the terms and conditions of the Creative Commons Attribution (CC BY) license (http://creativecommons.org/licenses/by/4.0/). 